BIOMEDICAL AND BIOSOCIAL ANTHROPOLOGY
$\begin{gathered}\text { Official Journal of the International Academy } \\ \text { of Integrative Anthropology } \\ \text { journal homepage: http://bba-journal.com }\end{gathered}$

\title{
Fractal dimension of the cortex and white matter of human cerebellum (magnetic resonance imaging study)
}

Maryenko N. I., Stepanenko O. Yu.

Kharkiv National Medical University, Kharkiv, Ukraine

\section{ARTICLE INFO}

Received: 20 August, 2020

Accepted: 22 September, 2020

UDC: $11: 57.086: 517: 530.191$

CORRESPONDING AUTHOR

e-mail: stepanenko@3g.ua Stepanenko O. Yu.

\begin{abstract}
The cerebellum is a typical structure with fractal properties, so fractal analysis is one of the main morphometric techniques that allow a comprehensive assessment of its morphofunctional state; the development of methods for differential measurement of the fractal dimension of various components of cerebellar tissue is necessary for complex morphological examination of the cerebellum using fractal analysis. The aim of the study was to develop an algorithm for differential fractal analysis and to determine the values of the fractal dimension of the cortex and white matter of human cerebellum using the study of magnetic resonance imaging scans. Digital T2 weighted magnetic resonance imaging scans of 30 conditionally healthy persons were used in the study. Fractal analysis of the distinct components of the cerebellar tissue was performed using the pixel dilation method. The fractal dimension values for all threshold brightness values (from 0 to 255) were determined. The confidence interval of the fractal dimension values based on the average values of the fractal dimension of the entire range of brightness values was calculated. Algorithms for image preprocessing were developed for an individual study of the different components of the cerebellum: cerebellar tissue as a whole, white matter, molecular and granular layers of the cortex. A differential fractal analysis technique has been developed to assess individual components of the cerebellar tissue. The values of the fractal dimension of white matter, granular and molecular layers of the cerebellar cortex were determined. The values of the fractal dimension of cerebellar tissue as a whole and the fractal dimension of the cerebellar cortex as a whole were the biggest. These values exceed the value of the fractal dimension of white matter. The average value of the fractal dimension (FD) for the threshold value of 80 (white matter) was 1.318 \pm 0.050 , for the value of 90 (white matter and the granular layer of the cortex) was $1.568 \pm 0.028$, for the value of 100 (cerebellar tissue as a whole) was $1.694 \pm 0.010$. The average FD of the granular layer of the cortex (brightness range 81-90) was $1.377 \pm 0.020$, the $F D$ of the molecular layer of the cortex (brightness range 91-100) was 1.353 \pm 0.020 , the average $F D$ of the cerebellar cortex as a whole (brightness range 81-100) was 1.564 \pm 0.018 . The obtained data can be used as diagnosis criteria to assess the morphofunctional state of the cerebellum using magnetic resonance imaging.
\end{abstract}

Keywords: fractal analysis, fractal dimension, pixel dilation, cerebellum, magnetic resonance imaging.

\section{Introduction}

Various neuroimaging methods are currently widely used to diagnose various pathological changes and diseases of the cerebellum. Magnetic resonance imaging (MRI) is the most commonly used neuroimaging method. The analysis of MRI scans takes into account both quantitative morphometric characteristics (linear size, area, volume) and qualitative characteristics (features of the shape and structure of various structures, the presence and features of pathological foci - necrosis, hemorrhages, tumors etc.) $[10,14]$.

The cerebellar tissue is not homogeneous and includes several components with different densities (white matter, granular and molecular layers of the cortex), which have different brightness of corresponding pixels on magnetic resonance images. Measurement of the volume and area (voxel-based morphometry) of various components of the 
cerebellar tissue (cortex and white matter) is widely used as a morphometric study in MRI [3, 4, 9, 11-13].

These morphometric studies revealed changes (decrease or increase) in the volume of gray and white matter of the cerebellar lobules in dyslexia [13], autism [3, $11,13]$, in children and adolescents with attention deficit hyperactivity disorder [9, 13], alcohol dependence [4], schizophrenia [15] and bipolar disorders [5].

Different algorithms of computer segmentation of digital images of the cerebellum are used for individual study of different structures of the cerebellum (determination of the boundary of the cortex and white matter, identification of different lobules) Those algorithms allow to determine the morphometric characteristics of different components and structures of the cerebellum $[16,18]$.

However, determining only the absolute and relative volumes of white and gray matter and their ratio does not take into account the spatial configuration of the cerebellum and relationships of different components of the cerebellar tissue.

The cerebellum is a typical structure with fractal properties, so fractal analysis is one of the main morphometric techniques that allow a comprehensive assessment of its morphofunctional state [1, 2, 6, 17].

The cerebellum has the heterogeneous (multifractal) structure. Thus, the development of methods for differential measurement of the fractal dimension of different components of cerebellar tissue is necessary for a complex morphological study of the cerebellum on magnetic resonance images using fractal analysis.

The aim of the study was to develop an algorithm for differential fractal analysis and to determine the values of the fractal dimension of the cortex and white matter of human cerebellum using the study of magnetic resonance imaging scans.

\section{Materials and methods}

The study was conducted in compliance with the basic bioethical provisions of the Council of Europe Convention on Human Rights and Biomedicine (04.04.1997), the Helsinki Declaration of the World Medical Association on the ethical principles of scientific medical research with human participation (1964-2008), and the order of Ministry of Health of Ukraine №690 dated 23.09.2009. The conclusion of the Commission on Ethics and Bioethics of Kharkiv National Medical University confirms that the study was conducted in compliance with human rights, in accordance with current legislation in Ukraine, meets international ethical requirements and does not violate ethical standards in science and standards of biomedical research (minutes of the meeting of the commission on ethics and bioethics of KhNMU №10 from 07.11.2018).

Magnetic resonance (MR) imaging scans of the brain were used in the study. MR images were obtained using a Siemens Magnetom Symphony tomograph with a magnetic induction value of $1.5 \mathrm{~T}$. Different components of cerebellar
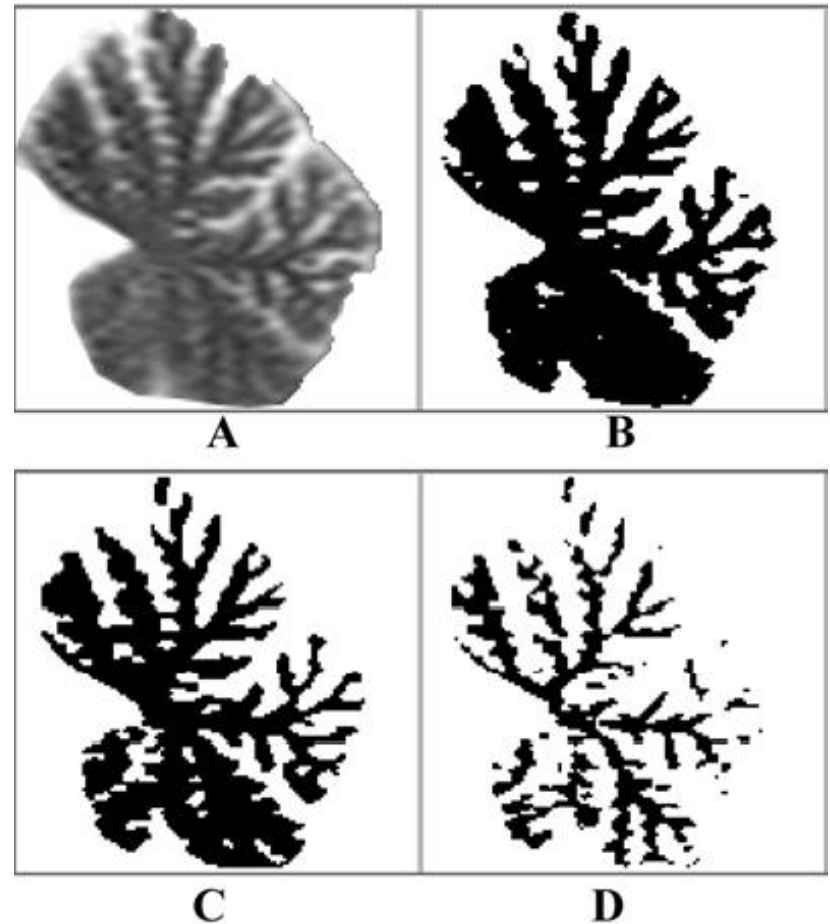

Fig. 1. Pre-processing (A) and segmentation (B-D) of MR images of the cerebellar vermis. Segmented pixels are colored black. B threshold 100, C - threshold 90, D - threshold 80.

tissue are more heterogeneous and contrasting on the T2weighted images then on the T1-weighted images, therefore T2-weighted images were used for the study.

The study used tomograms of 30 conditionally healthy persons (without structural changes in the brain) aged 1830 years (15 men and 15 women).

A digital image with a resolution of 128 pixels per inch and a size of $128 \times 128$ pixels was created in Adobe Photoshop CS5 for image processing and subsequent measurements. One of the parasagittal sections of the brain containing the tomographic section of the cerebellum was selected and the fragment was copied using the MR tomogram viewer "Syngo Fast View". This fragment was placed in the created digital image so that the section of the cerebellum filled the area of the square. Then the surrounding areas were removed from the image: those areas of the image that do not contain a section of the cerebellum were selected and filled with white color (Fig. 1, A).

This was followed by image segmentation using the Adobe Photoshop CS5 "threshold" tool. Segmentation was performed depending on the brightness of the pixels corresponding to different components of the cerebellar tissue. All pixels darker than the brightness threshold were colored black, lighter than the brightness threshold were colored white. Images were segmented by all theoretically possible brightness thresholds - from 0 to 255 (see Fig. 1, A, B, C). For example, all pixels with brightness values from 0 to 80 inclusive were segmented for a brightness value of 80 . 
Fractal dimension (FD) was measured on segmented images. Fractal dimensions of various components of cerebellar tissue that have a density equal to or greater than a certain threshold value were determined. The method of pixel dilatation in the author's modification described earlier was used for fractal analysis [7].

Statistical data processing was performed using Excel 2010. The arithmetic mean (M) and its standard error (mM) were calculated, and the confidence interval of values as $M \pm 2 \sigma$ was determined. Pearson's correlation coefficient was calculated to determine the relationship between the values. The significance of statistical differences was assessed using the Mann-Whitney test. The significance of the correlation was assessed using Student's T test.

\section{Results}

The arithmetic means of the fractal dimension values for the entire range of brightness values (from 0 to 255) were calculated as a result of study of MRI scans of 30 persons (Fig. 2).

The confidence interval of $F D$ values as $M \pm 2 \sigma$ was determined based on the arithmetic mean (M) and standard deviation $(\sigma)$ values for the entire range of brightness thresholds used for segmentation (see Fig. 2).

The values of FD depend on the brightness, and this dependence is close to the logarithmic function, as can be seen from Fig. 2. FD increases mainly in the range of brightness values from 70 to 100 .

The cerebellar tissue is not homogeneous, which causes the heterogeneity of the brightness of the pixels of digital MR images. Different components of the cerebellar tissue have different densities and, consequently, different values of the brightness of the pixels of the digital image. If we compare the result of segmentation of the same original image (see Fig. 1, A), segmented by different levels of brightness (see Fig. 1, B-D), we can see that the different components of the cerebellar tissue are segmented on the resulting images. The lower the threshold level of brightness for segmentation is, the bigger density of the visualized structure is.

The brightness thresholds for segmentation of the

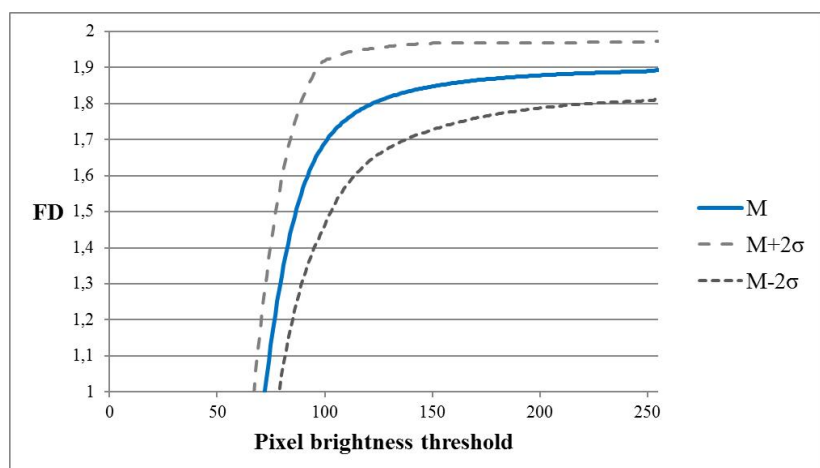

Fig. 2. Cerebellum fractal dimension values depending on the pixel brightness threshold used for segmentation: arithmetic mean (M) and confidence interval $(\mathrm{M} \pm 2 \sigma)$.

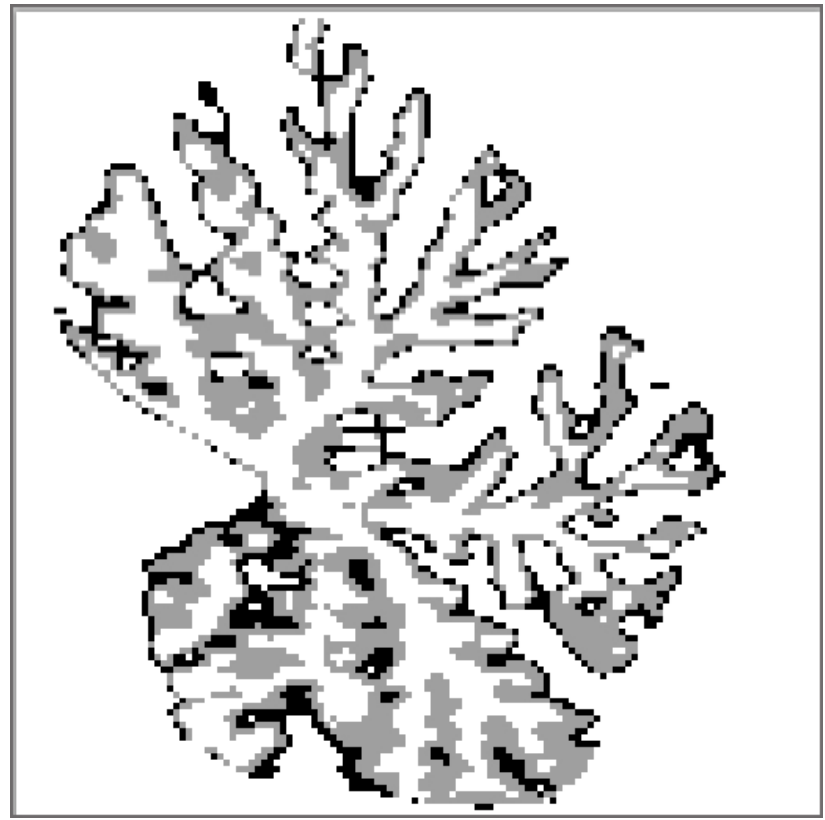

Fig. 3. Segmented components of the cerebellar tissue: white matter (segmentation threshold 80 ) is colored white, the granular layer of the cortex (difference of segmentation thresholds 91-80) is colored gray, the molecular layer of the cortex (difference of segmentation thresholds $91-100$ ) is colored black.

individual components of the cerebellar tissue were determined by visual comparison and also based on the results of cluster analysis of the fractal dimension of the cerebellum, which was carried out in our previous studies [8].

Thus, the white matter corresponds to the range of brightness values from 0 to 80 (see Fig. 1, D), the granular layer of the cortex - 81-90 (see Fig. 1, C), the molecular layer of the cortex - 91-100 (see Fig. 1, B). The brightness threshold 80 , used to segment the digital image, separates only the white matter of the cerebellum from the background, 90 white matter with a granular layer of cortex, 100 - white matter, granular and molecular layers of the cortex (cerebellar tissue as a whole) (Fig. 3).

The average value of the fractal dimension for the threshold value of 80 (white matter) was $1.318 \pm 0.050$, for the value of 90 (white matter and the granular layer of the cortex) was $1.568 \pm 0.028$, for the value of 100 (cerebellar tissue as a whole) was $1.694 \pm 0.010$. The average FD of the granular layer of the cortex (brightness range 81-90) was $1.377 \pm 0.020$, the FD of the molecular layer of the cortex (brightness range 91-100) was $1.353 \pm 0.020$, the average FD of the cerebellar cortex as a whole (brightness range 81100 ) was $1.564 \pm 0.018$. The distribution of these values is shown in Fig. 4.

Correlation relationships between the FD values of different components of the cerebellar tissue were determined using calculation of the Pearson's correlation coefficient (r) (Fig. 5). The strength and direction of the correlation relationships were different.

FD of the cerebellar white matter has a significant 


\begin{tabular}{|l|c|c|c|c|c|c|}
\hline \multicolumn{2}{|c|}{$\begin{aligned} 2,000 \\
1,900\end{aligned}$} \\
1,800
\end{tabular}

Fig. 4. The distribution of values of the fractal dimensions of the different components of cerebellar tissue.

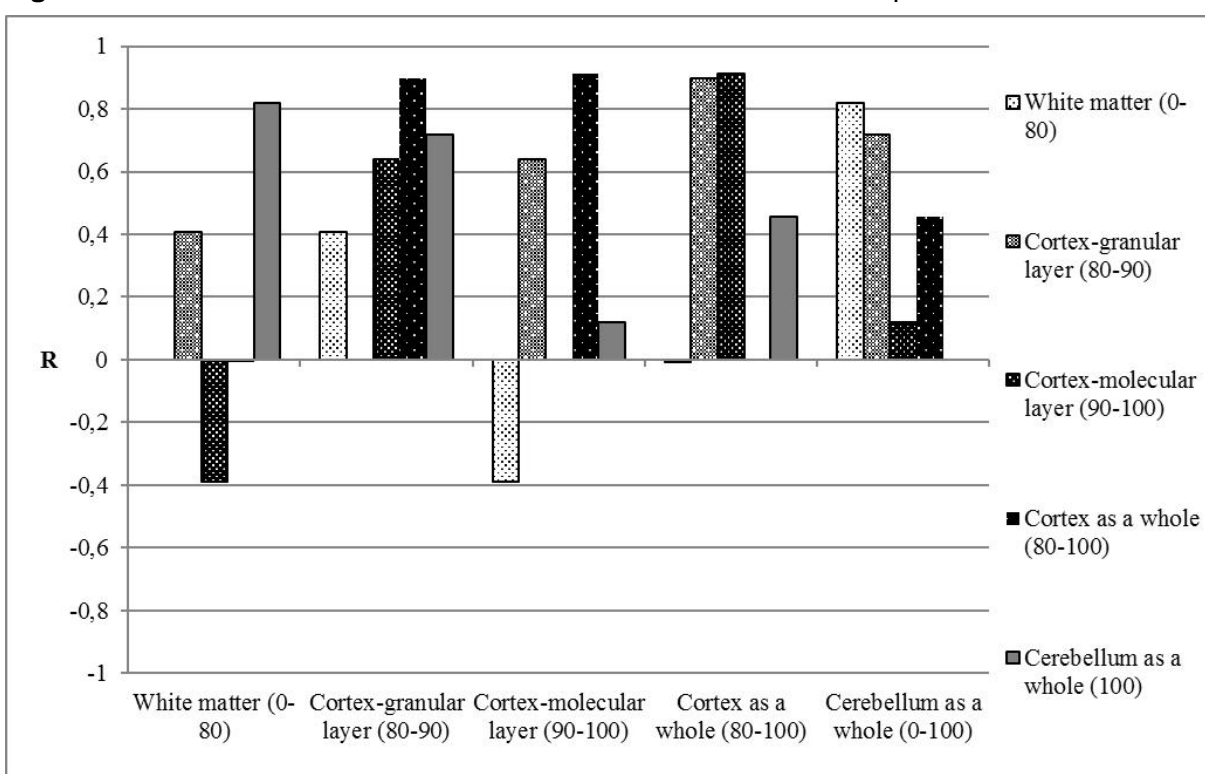

Fig. 5. The correlation relationships of the fractal dimensions of the main components of cerebellar tissue.

with the FD of the granular layer, but has negative linear relationship with the FD of the molecular layer. The FD of the cerebellar cortex as a whole has a significant strong positive linear relationship with FD of the molecular layer $(r=0.91, p=0.000)$ as well as with FD of the granular layer $(r=0.90, p=0.000)$. The FD of the molecular layer has a greater effect on the FD of the cortex as a whole than the FD of the granular layer, because the molecular layer forms the outer contour of the cerebellum. There is a significant strong positive linear relationship between fractal dimensions of the molecular and granular layers of the cerebellar cortex $(r=0.64$, $p=0.000)$.

FD of cerebellar tissue as a whole has a significant strong positive linear relationship with FD of white matter $(r=0.82, p=0.000)$ as well as with FD of the granular layer $(r=0.72, p=0.000)$. These components of the cerebellar tissue have a high variability of structure and make up a significant part of the volume of the cerebellum. FD of cerebellar tissue as a whole also has a significant moderate positive linear relationship with FD of the cortex as a whole $(r=0.46$, $\mathrm{p}=0.008$ ) and has no significant relationship with the FD of the molecular layer of the cortex $(r=0.12, p=0.833)$.

The values of the fractal dimensions of different moderate positive linear relationship with FD of the granular layer of the cortex $(r=0.41, p=0.017)$, significant moderate negative linear relationship with FD of the molecular layer $(r=-0.39, p=0.023)$; but it has no significant relationship with FD of the cerebellar cortex as a whole $(r=-0.01, p=0.998)$. This feature is associated with the peculiarities of the structure of the cerebellum at the microanatomical level. The structure of the granular layer of the cerebellar cortex is more variable. This layer covers the white matter on the outside, duplicating its outer contour. The structure of the molecular layer of the cerebellar cortex is less variable: this layer has less thickness variability than the granular layer. Thus, the FD of white matter has a positive linear relationship components of the cerebellar tissue in men and women were also determined (Fig. 6). It was found that the values of the fractal dimensions of the white matter of the cerebellum, cerebellar cortex as a whole, granular and molecular layers of the cortex and cerebellar tissue as a whole in men and women do not have significant difference $(p<0.001)$.

\section{Discussion}

The studies on fractal analysis of the cerebellum in the scientific literature in general are not numerous $[1,2,6$, 17]. The values of the fractal dimensions of the white matter and cortex of the cerebellum were determined differentially 


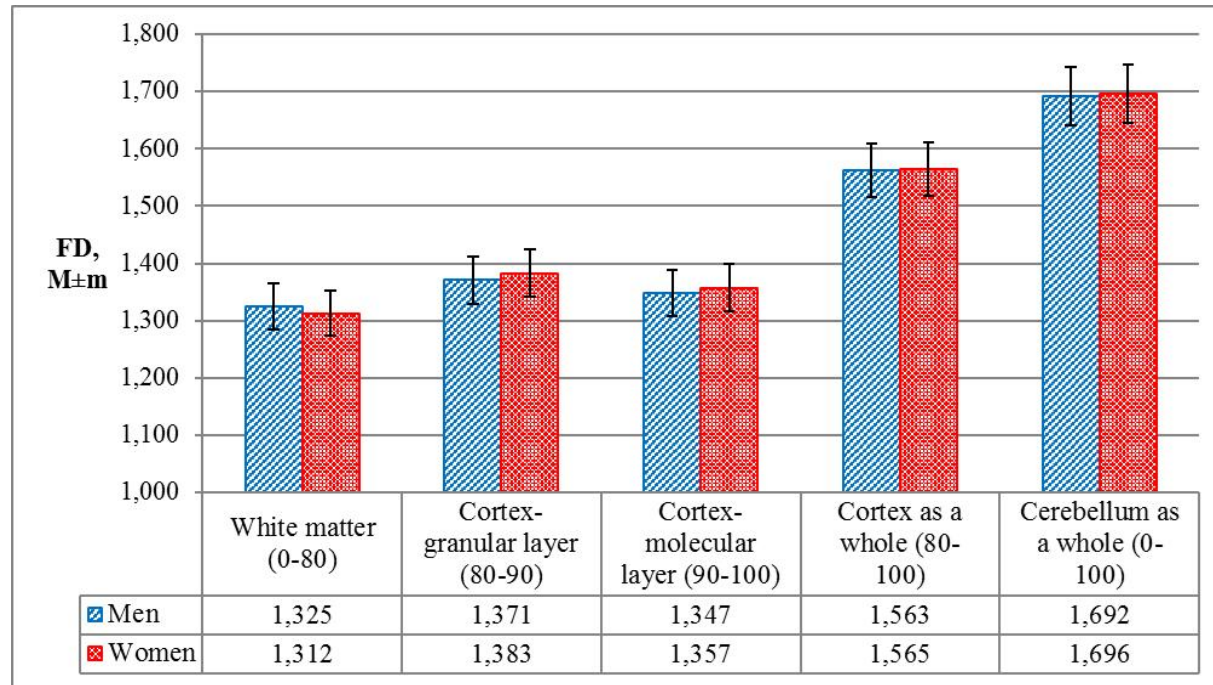

Fig.6. The values of the fractal dimensions of the main components of cerebellar tissue in men and women.

in some studies. Two-dimensional (2D) and threedimensional (3D) fractal dimensions were determined in different studies. Thus, the 2D fractal dimension values of two-dimensional images of the cerebellum were determined using the box counting method. The MRI scans of 16 conditionally healthy persons were investigated. The average FD value of the white matter was $1.49 \pm 0.06$ and the average FD value of the gray matter was $1.56 \pm 0.05$. FD values of the different layers of the cerebellar cortex were not determined individually [1]. These data are generally consistent with data obtained in the present study: the FD value of gray matter exceeds the FD value of white matter. The difference between the average values of fractal dimension obtained in the study [1] and in the present study may be due to the difference in the number of studied objects, differences in algorithms of the image preprocessing and segmentation and difference in methods of fractal analysis (box counting and pixel dilation, respectively). In other works, the values of 3D fractal dimension of the white and gray matter of the cerebellum were determined $[2,17]$. The average values of $3 D$ fractal

\section{References}

[1] Akar, E., Kara, S., Akdemir, H., \& Kiris, A. (2015). Fractal dimension analysis of cerebellum in Chiari Malformation type I. Computers in biology and medicine, 64, 179-186. doi: 10.1016/ j.compbiomed.2015.06.024

[2] Akar, E., Kara, S., Akdemir, H., \& Kiris, A. (2017). 3D structural complexity analysis of cerebellum in Chiari malformation type I. Medical \& biological engineering \& computing, 55(12), 2169-2182. doi: 10.1007/s11517-017-1661-7

[3] D'Mello, A. M., Crocetti, D., Mostofsky, S. H., \& Stoodley, C. J. (2015). Cerebellar gray matter and lobular volumes correlate with core autism symptoms. Neurolmage. Clinical, 7, 631639. doi: 10.1016/j.nicl.2015.02.007

[4] Hill, S. Y., Wang, S., Carter, H., Tessner, K., Holmes, B., McDermott, M., Zezza, N., \& Stiffler, S. (2011). Cerebellum volume in high-risk offspring from multiplex alcohol dependence families: association with allelic variation in dimension of the white matter were $2.26 \pm 0.05$ [2] and $2.2746 \pm 0.0446$ [17] and the average values $3 \mathrm{D}$ fractal dimension of the gray matter were $2.49 \pm 0.04$ [2] and 2.5267 \pm 0.0228 [17]. Fractal dimensions of different layers of the cerebellar cortex also were not determined individually. In the studies [2, 17] the FD values of the gray matter also exceed the corresponding values of the white matter, which corresponds to results of the present study. The values of 3D fractal dimension exceed the values obtained in the present study, because the threedimensional FD can vary from 2 to 3 and theoretically always exceeds the two-dimensional FD, which has a value from 1 to 2 .

The obtained data can be used as diagnosis criteria to assess the morphofunctional state of the cerebellum using magnetic resonance imaging.

\section{Conclusions}

1. A technique of differential fractal analysis for assessment of individual components of cerebellar tissue has been developed.

2. Algorithms of image pre-processing for the individual study of the cerebellar tissue as a whole, white matter and individual layers of the cerebellar cortex were determined.

3 . The values of fractal dimension of white matter, granular and molecular layers of the cerebellar cortex were determined. The fractal dimension of the cerebellar tissue as a whole and the fractal dimension of the cortex as a whole have the biggest values, which exceed the value of the fractal dimension of cerebellar white matter.

GABRA2 and BDNF. Psychiatry research, 194(3), 304-313. doi: 10.1016/j.pscychresns.2011.05.006

[5] Johnson, C. P., Christensen, G. E., Fiedorowicz, J. G., Mani, M., Shaffer, J. J. Jr., Magnotta, V. A., \& Wemmie, J. A. (2018). Alterations of the cerebellum and basal ganglia in bipolar disorder mood states detected by quantitative T1 $\rho$ mapping. Bipolar disorders, 20(4), 381-390. doi: 10.1111/bdi.12581

[6] Liu, J. Z., Zhang, L. D., \& Yue, G. H. (2003). Fractal dimension in human cerebellum measured by magnetic resonance imaging. Biophysical journal, 85(6), 4041-4046. doi: 10.1016/S00063495(03)74817-6

[7] Maryenko, N. I., Stepanenko, O. Yu. (2019). Фрактальний аналіз як морфометричний метод в морфології: спосіб дилатації пікселів при дослідженні цифрових зображень анатомічних структур [Fractal analysis as a morphometric method in morphology: a pixel dilatation technique in the study of digital 
images of anatomical structures]. Український журнал медицини, біології та спорту - Medicine today and tomorrow, 1(82), 8-14. doi: 10.35339/msz.2019.82.01.02

[8] Maryenko, N. I. (2020). Кластерний аналіз фрактальної розмірності мозочка людини [Cluster Analysis of Human Cerebellum Fractal Dimension]. Медицина сьогодні і завтра - Ukr. z. med. biol. sportu, 5(5), 66-72. doi: 10.26693/ jmbs05.05.066

[9] Montes, L. G., Ricardo-Garcell, J., De la Torre, L. B., Alcantara, H. P., Garcia, R. B., Acosta, D. A., \& Bouzas, A. F. (2011). Cerebellar gray matter density in females with ADHD combined type: a cross-sectional voxel-based morphometry study. Journal of attention disorders, 15(5), 368-381. doi: 10.1177/ 1087054710366421

[10] Park, M. T., Pipitone, J., Baer, L. H., Winterburn, J. L., Shah, Y., Chavez, S., ... \& Chakravarty, M. M. (2014). Derivation of high-resolution MRI atlases of the human cerebellum at $3 \mathrm{~T}$ and segmentation using multiple automatically generated templates. Neurolmage, 95, 217-231. doi: 10.1016/ j.neuroimage.2014.03.037

[11] Riva, D., Annunziata, S., Contarino, V., Erbetta, A., Aquino, D., \& Bulgheroni, S. (2013). Gray matter reduction in the vermis and CRUS-II is associated with social and interaction deficits in low-functioning children with autistic spectrum disorders: a VBM-DARTEL Study. Cerebellum (London, England), 12(5), 676-685. doi: 10.1007/s12311-013-0469-8

[12] Spulber, G., Niskanen, E., Macdonald, S., Kivipelto, M., Padilla, D. F., Julkunen, V., ... \& Soininen, H. (2012). Evolution of global and local grey matter atrophy on serial MRI scans during the progression from $\mathrm{MCl}$ to $\mathrm{AD}$. Current Alzheimer research,
9(4), 516-524. doi: 10.2174/156720512800492486

[13] Stoodley C. J. (2014). Distinct regions of the cerebellum show gray matter decreases in autism, ADHD, and developmental dyslexia. Frontiers in systems neuroscience, 8, 92. doi: 10.3389/fnsys.2014.00092

[14] van Baarsen, K. M., Kleinnijenhuis, M., Jbabdi, S., Sotiropoulos, S. N., Grotenhuis, J. A., \& van Cappellen van Walsum, A. M. (2016). A probabilistic atlas of the cerebellar white matter. Neurolmage, 124(Pt A), 724-732. doi: 10.1016/ j.neuroimage.2015.09.014

[15] Wang, J., Zhou, L., Cui, C., Liu, Z., \& Lu, J. (2017). Gray matter morphological anomalies in the cerebellar vermis in firstepisode schizophrenia patients with cognitive deficits. BMC psychiatry, 17(1), 374. doi: 10.1186/s12888-017-1543-4

[16] Weier, K., Fonov, V., Lavoie, K., Doyon, J., \& Collins, D. L. (2014). Rapid automatic segmentation of the human cerebellum and its lobules (RASCAL)--implementation and application of the patch-based label-fusion technique with a template library to segment the human cerebellum. Human brain mapping, 35(10), 5026-5039. doi: 10.1002/hbm.22529

[17] Wu, Y. T., Shyu, K. K., Jao, C. W., Wang, Z. Y., Soong, B. W., Wu, H. M., \& Wang, P. S. (2010). Fractal dimension analysis for quantifying cerebellar morphological change of multiple system atrophy of the cerebellar type (MSA-C). Neurolmage, 49(1), 539-551. doi: 10.1016/j.neuroimage.2009.07.042

[18] Yang, Z., Ye, C., Bogovic, J. A., Carass, A., Jedynak, B. M., Ying, S. H., \& Prince, J. L. (2016). Automated cerebellar lobule segmentation with application to cerebellar structural analysis in cerebellar disease. Neurolmage, 127, 435-444. doi: 10.1016/ j.neuroimage.2015.09.032 